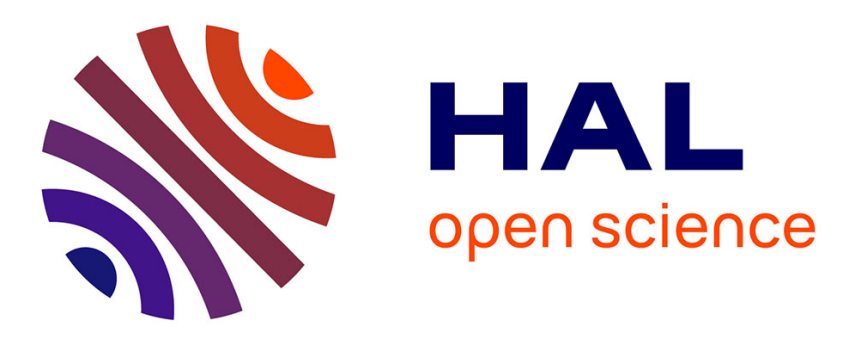

\title{
Benchmarking Change Detection in Urban 3D Point Clouds
}

Iris de Gélis, Sébastien Lefèvre, Thomas Corpetti, Thomas Ristorcelli, Chloé Thénoz, Pierre Lassalle

\section{- To cite this version:}

Iris de Gélis, Sébastien Lefèvre, Thomas Corpetti, Thomas Ristorcelli, Chloé Thénoz, et al.. Benchmarking Change Detection in Urban 3D Point Clouds. IGARSS 2021 - IEEE International Geoscience and Remote Sensing Symposium, Jul 2021, Brussels, Belgium. hal-03379985

\section{HAL Id: hal-03379985 \\ https://hal.science/hal-03379985}

Submitted on 15 Oct 2021

HAL is a multi-disciplinary open access archive for the deposit and dissemination of scientific research documents, whether they are published or not. The documents may come from teaching and research institutions in France or abroad, or from public or private research centers.
L'archive ouverte pluridisciplinaire HAL, est destinée au dépôt et à la diffusion de documents scientifiques de niveau recherche, publiés ou non, émanant des établissements d'enseignement et de recherche français ou étrangers, des laboratoires publics ou privés. 


\title{
BENCHMARKING CHANGE DETECTION IN URBAN 3D POINT CLOUDS
}

\author{
Iris de Gélis ${ }^{1,2}$, Sébastien Lefèvre ${ }^{2}$, Thomas Corpetti ${ }^{3}$, Thomas Ristorcelli ${ }^{1}$, Chloé Thénoz ${ }^{1}$, Pierre Lassalle ${ }^{4}$ \\ ${ }^{1}$ Magellium, F-31000 Toulouse, France \\ ${ }^{2}$ Université Bretagne-Sud, IRISA UMR 6074, F-56000 Vannes, France \\ ${ }^{3}$ CNRS, LETG UMR 6554, F-35000 Rennes, France \\ ${ }^{4}$ CNES, F-31000 Toulouse, France
}

\begin{abstract}
According to the United Nations, $70 \%$ of earth population is going to live in cities by 2050 . Given this fast urban evolution, urban monitoring is a key process to qualify sustainable development. Vertical changes need to be assessed, and various methods for 3D change detection have been published. However, there is no common quantitative benchmark assessing their performance in urban areas yet. In this paper, we aim to fill this gap and introduce a simulation tool to generate synthetic 3D point cloud data in a well-controlled scenario. These data are then used to compare qualitatively and quantitatively representative 3D change detection methods for urban areas. These methods are based on distance computation (DSMd, C2C, M3C2), traditional machine learning (RF with stability feature) and deep learning (Feed Forward and Siamese networks). We distinguish between binary and multi-class classification of changes at different levels (3D points, 2D pixels, and 2D patches). While deep neural networks have led to numerous success in remote sensing, we show that they do not systematically outperform more simple methods for 3D change detection. Besides, the existing networks are limited to 2D patches while outputs at the pixel or point scale are more attractive.
\end{abstract}

Index Terms - 3D change detection, urban monitoring, bi-temporal point clouds dataset, LiDAR simulator

\section{INTRODUCTION}

Change detection (CD) in urban areas is an important field of study in remote sensing since it provides key information regarding urbanization of our planet. Multiple types of data could be used for $\mathrm{CD}$ including $3 \mathrm{D}$ point clouds (PCs). While a lot of existing studies use 2D images for CD, 3D PCs bring some supplementary information on height that seems useful in the context of building change extraction as main modifications occur on the height axis. Furthermore, spectral variability of a same object over time, difference of viewing angles between acquisition of 2D images, perspective and distortion effects could complicate CD retrieval based on 2D data [1].

I. de Gélis work is funded by Magellium and supported by a CNES grant.
While CD on urban 3D data has been addressed in several studies, so far there is a strong lack of experimental comparison. To the best of our knowledge, the only comparative analysis [2] remains at a qualitative level and does not include deep learning (DL) methods that represent the state-of-the-art in remote sensing. In this paper, we aim to fill this gap through two contributions: first we design a novel simulator of 3D aerial LiDAR PCs over urban areas that has been specifically tailored to enable the introduction of changes over buildings, leading to generation of annotated 3D CD urban datasets in well-controlled benchmarking scenarios. We then provide a comparison between representative methods of the state-ofthe-art in the context of Aerial LiDAR Survey (ALS) in urban areas, from classical distance based methods to the latest deep learning developments.

\section{3D CHANGE DETECTION METHODS}

We present here the methods that have been included in our experimental comparison. They have been selected as representative of the current literature, and are based on distance computation, machine learning (ML) with hand-crafted features or DL. In order to process 3D PCs, two main strategies have been reported, namely converting 3D PCs into Digital Surface Models (DSMs) or directly processing raw PCs. A DSM is a 2D rasterisation of the PCs which allows us to have for each pixel some information on height. Often inspired by $\mathrm{CD}$ algorithms based on 2D images, these methods are easier to apply as DSMs can be seen as grayscale images. Apart from the difficulty of processing unordered and sparse data like PCs, methods operating on raw PCs have the advantage of avoiding the loss of information brought by the rasterisation process.

\subsection{Distance-based methods}

The first idea to detect $3 \mathrm{D}$ changes and the easiest one is to measure difference between DSMs. It was first explored in [3] for building change extraction. It is still often used because of its simplicity and of the quality of results for buildings 
change highlighting, and even in other fields of earth observation [4]. After having computed the DSM difference (DSMd), a thresholding is required to retrieve changes. One can either choose an empiric threshold [3] or use the Otsu algorithm in order to select thresholds according to the DSMd histogram. Finally, DSMd is often the first step in a lot of studies, some further refinement of results is usually done to obtain better results. For instance, morphological filters such as opening can be apply to filter isolated pixels [5].

Distance computation can also be done directly on 3D PCs as in C2C (cloud to cloud) method [6] where PCs are subdivided in octrees and the Hausdorff distance is used. In [7], authors first extract surface normal and orientation at specific scales with the local surface roughness. The measurement of the mean surface change is performed along the normal direction is realised (method M3C2: Multi Scale Model to Model Cloud Comparison). $\mathrm{M} 3 \mathrm{C} 2$ is able to differentiate positive and negative changes conversely to the $\mathrm{C} 2 \mathrm{C}$, and comes with a lower computation time [2]. A threshold can be apply on results of both methods to categorize each point into classes.

\subsection{Machine learning with hand-crafted features}

Several studies, called post-classification methods, consist first of classifying the data at each date, before comparing the classification results. On the contrary, [8] tries to combine classification and change detection in one single step. To do so, the authors extract features related to points distribution, terrain elevation, multi-target capability of LiDAR and finally a feature called stability. The stability is computed for each point based on the distribution of the neighboring points in the PC coming from the other date, and more precisely as the ratio of the number of points in a spherical and vertical cylindrical z-oriented neighborhood. Then, a random forest (RF) algorithm is applied to obtain a supervised classification of changes. This method will be noted RF in our experiments.

\subsection{Deep learning methods}

More recently, [9] tries to use DL on DSMs to detect changes in patches in urban areas. Two kind of Convolutional Neural Network (CNN) are explored: a Feed-Forward (FF) network and a Siamese architecture. Siamese networks are often used for change detection or similarity computation between two inputs, and have been largely used for remote sensing application. They are known to provide high-accuracy results even with significantly heterogeneous inputs. The objective of [9] is to use bi-temporal multi-modal 3D information from both ALS and photogrammetric PCs. They achieve quite good results with a precision of $63 \%$ for both FF and Siamese networks on DSM only, where DSMd reached only $38 \%$ according to the original paper. Let us notice that DSMd is here measured per patch, i.e. an average of DSMd is made over the whole patch and thresholded to distinguish between changed or unchanged patches. In their study, [9] consider only a binary classification of patches. We extended their architectures in order to be able to predict the three classes of interest: unchanged, construction and demolition.

\section{BENCHMARKING URBAN 3D CHANGES}

To assess the methods reviewed in the previous section, we rely on a data simulation tool that allows us to build a novel urban change dataset. We present here these different components together with the evaluation metrics used in our study to compare the different methods.

\subsection{Point cloud simulator}

In order to perform quantitative evaluation of various methods, we have designed a simulator of LiDAR surveying over urban areas to allow us a fine characterization of the behaviour of 3D change detection methods. Given a 3D model (OBJ format) of a city, the simulator allows us to introduce random changes in the model and generate a synthetic ALS above the city. With adding or removing buildings in the model, we can simulate construction or demolition of buildings. The simulator allows us to obtain as many 3D PCs over urban changed areas as needed. It could be useful especially for DL supervised approaches that require a lot of training instances. Moreover, the created PCs are all directly annotated by the simulator according to the changes, thus no time-consuming manual annotation needs to be done on these data. Figure 1 gives an example of PCs at two epochs generated by the simulator. The second epoch is labelled according to the changes.

A flight plan is defined according to predefined parameters such as resolution, covering between swaths and scan angle. For each obtained model, the ALS simulation is realised thanks to the flight plan and ray tracing with the Visualisation ToolKit (VTK) python library ${ }^{1}$. Finally, Gaussian noise can be added to the simulation to simulate errors and lack of precision in LiDAR range measuring and scan direction. A qualitative validation of PCs acquired thanks to the simulator has been realised by an expert of 3D PCs.

\subsection{Urban change dataset}

We use our simulator to generate PC synthetic data from a 3D Level of Detail 2 (LoD2) model of the french city of Lyon ${ }^{2}$. Two dates are considered with several building construction and demolition instances. ${ }^{3}$

In practice the flight plan is set randomly. As a consequence, the swath will not be the same between pairs of PCs,

\footnotetext{
${ }^{1}$ https://vtk.org

${ }^{2}$ https://geo.data.gouv. fr/datasets/073198934974 $2867 \mathrm{f} 8 \mathrm{e} 659 \mathrm{~b} 4 \mathrm{~d} 70 \mathrm{~b} 707612$ bece 89

${ }^{3}$ The dataset is publicly available at the following link: https: / / ieee -dataport.org/open-access/urb3dcd-urban-point-clou ds-simulated-dataset-3d-change-detection
} 


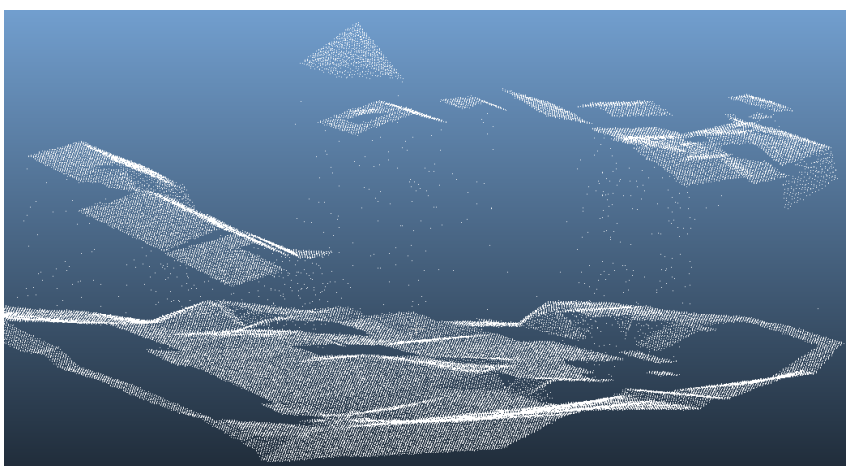

(a) Epoch 1

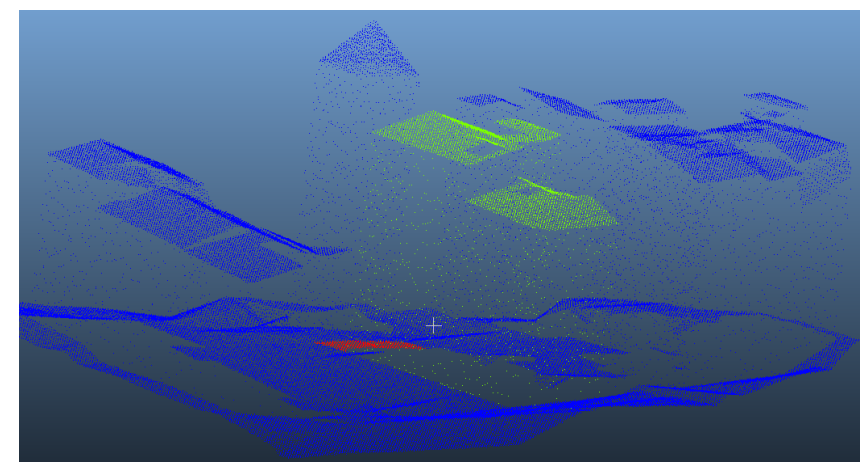

(b) Epoch 2

Fig. 1. Sample PCs at two epochs, with new buildings (green), demolished buildings (red), and unchanged objects (blue) in (b).

thus each acquisition may not have exactly the same visible or invisible parts. Moreover range error is set to $5 \mathrm{~cm}$ and scan direction error across track to $0.01^{\circ}$ and the average resolution is 0.5 points $/ \mathrm{m}^{2}$. By doing so, we ensure more realistic and tricky simulation conditions, in particular with this challenging low spatial resolution.

We have split the area into three distinct parts to provide a common testing set for all methods, and some specific training/validation sets for ML/DL-based methods. Figure 2 presents a vertical view of the LoD2 model of Lyon where all available buildings are shown (height shown in color).

A large number of PC pairs has been used, building construction/destruction between the two dates being randomly generated by the simulator. It could be seen as a data augmentation process especially useful for supervised learning.

\subsection{Evaluation metrics protocol}

The quantitative evaluation relies on the mean of Intersection over Union (IoU) over classes of change. We recall that for a given class, IoU is defined by the ratio $\mathrm{TP} /(\mathrm{TP}+\mathrm{FP}+\mathrm{FN})$ where TP, FP and FN stand for True Positive, False Positive and False Negative, respectively. In case of binary classification, the formula is used as it is with positive meaning change. Otherwise, the mean of IoU over both change classes (construction and demolition) is used. This metric has been preferred over accuracy or precision measures since it allows us to focus on classes of change, while most of pixels or points remain unchanged between the two epochs.

\section{RESULTS AND DISCUSSION}

Experimental results are summarized in Table 1, considering the three output levels (3D point, 2D pixel and 2D patch) as well as binary and multiclass change prediction.

We can observe that among methods that process directly 3D PCs, best results are achieved with RF [8], that however requires some level of supervision and the prior extraction of

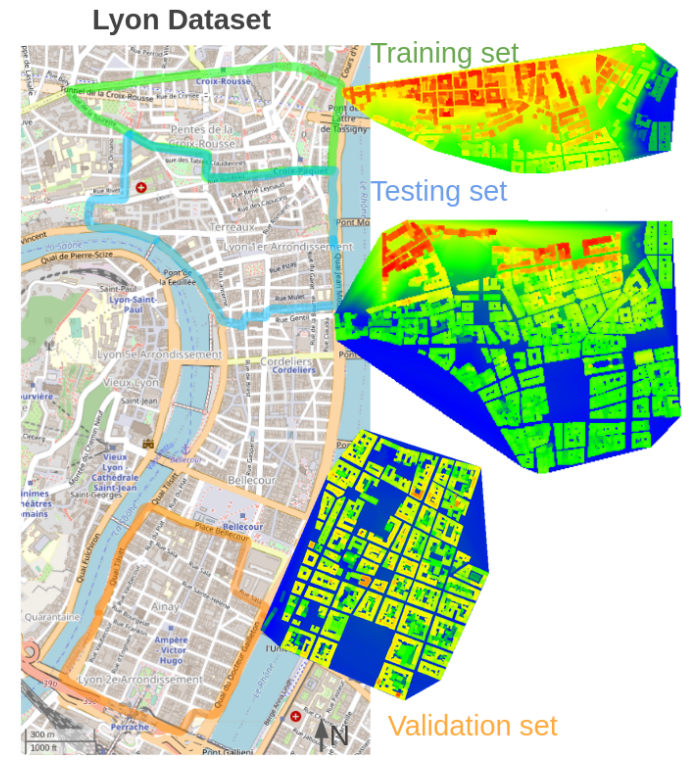

Fig. 2. Lyon dataset split into 3 distinct parts: West and North are used for training and validation for DL and ML methods, South is always the testing set. Height is shown in color.

hand-crafted features. In our dataset, we have changed the LiDAR flight plan between the two dates, thus the point of view is not exactly the same. These varying positions of the LiDAR during the acquisition step introduce different hidden parts in PCs especially in dense urban areas due to building shadows for instance. As a consequence, classification errors are mainly due to this effect whatever the method. Let us note that the stability feature, by taking into account the neighborhood of each point, allows RF to bring more precision in the prediction of change classes.

When comparing predictions operating at the pixel level, we can notice the positive effect of the morphological opening. Indeed, we are interested here in changes related to building, and building are never isolated pixels. We also report the 


\begin{tabular}{|c|c|c|c|c|c|c|c|c|c|}
\hline & \multirow[t]{2}{*}{ Name } & \multirow[t]{2}{*}{ Type } & \multicolumn{2}{|c|}{ Points 3D } & \multicolumn{2}{|c|}{ Pixel 2D } & \multicolumn{2}{|c|}{ Patch 2D } & \multirow[t]{2}{*}{ Ref. } \\
\hline & & & Binary & $\mathrm{MC}$ & Binary & $\mathrm{MC}$ & Binary & $\mathrm{MC}$ & \\
\hline 1a & DSMd + Empiric thresholding & Dist & - & - & 0.37 & 0.37 & 0.36 & 0.35 & {$[3]$} \\
\hline $1 b$ & DSMd + Otsu & Dist & - & - & 0.59 & 0.59 & 0.62 & 0.60 & [3] \\
\hline $1 \mathrm{c}$ & DSMd + Otsu + Opening & Dist & - & - & 0.72 & 0.72 & 0.82 & 0.80 & {$[3],[5]$} \\
\hline 2 & C2C Empiric & Dist & 0.50 & - & - & - & - & - & [6] \\
\hline 3 & M3C2 Empiric & Dist & 0.34 & 0.30 & - & - & - & - & [7] \\
\hline 4 & RF & ML & 0.64 & 0.63 & - & - & - & - & [8] \\
\hline 5 & FF & DL & - & - & - & - & 0.78 & 0.75 & [9] \\
\hline 6 & Siam. & DL & - & - & - & - & 0.72 & 0.64 & [9] \\
\hline
\end{tabular}

Table 1. Mean of IoU on change class. MC stands for multi-class classification.

improvement brought by Otsu thresholding.

Finally, for methods dealing with image patches, as done in [9], we extend the DSMd method to patch prediction by attributing a label of change to patches where the percentage of pixel labeled as changed after the thresholding, and eventually here the opening, is higher than $10 \%$. The same process is made in order to build the ground truth database for the learning step of DL methods. As observed in [9], DL methods outperform DSMd with Otsu thresholding. However, simply filtering the latter with a morphological opening retains wider changes, leading to even higher results. This certainly calls for developing more spatially-aware deep neural networks for urban change detection, able to output pixel or point-level results.

\section{CONCLUSION}

In this paper, we have dealt with 3D urban change detection and provided a novel benchmark to assess state-of-theart methods. It relies on an original simulator tool to generate synthetic point clouds in a well-controlled experimental protocol (in particular spatial resolution, flight plan and noise), yielding training and evaluations databases without requiring a costly manual labeling at the point level.

We have then compared representative methods, based on distance computation, machine learning with handcraft features, and more recent deep learning ones. Their results can be assessed at three different scales, in 3D as points, or in 2D as pixels or patches, and in a binary classification or multiclass scenario distinguishing new and demolished buildings.

Urban 3D change detection is still far from benefiting from the advances of deep learning conversely to other EO applications. This might be explained by the lack of labeled datasets and too simple network architectures unable to cope with changes at the point level. While our contribution tackles the former, our future work will focus on the latter.

\section{REFERENCES}

[1] R. Qin, J. Tian, and P. Reinartz, "3D change detection approaches and applications," $P \& R S$, vol. 122, pp. 4156, 2016.
[2] S. Shirowzhan, S.M.E Sepasgozar, H. Li, J. Trinder, and P. Tang, "Comparative analysis of machine learning and point-based algorithms for detecting $3 \mathrm{~d}$ changes in buildings over time using bi-temporal lidar data," Automation in Construction, vol. 105, pp. 102841, 2019.

[3] H. Murakami, K. Nakagawa, H. Hasegawa, T. Shibata, and E. Iwanami, "Change detection of buildings using an airborne laser scanner," $P \& R S$, vol. 54, no. 2-3, pp. 148-152, 1999.

[4] U. Okyay, J. Telling, C.L. Glennie, and W.E. Dietrich, "Airborne lidar change detection: An overview of earth sciences applications," Earth-Science Reviews, vol. 198, pp. 102929, 2019.

[5] C. Stal, F. Tack, P. De Maeyer, A. De Wulf, and R. Goossens, "Airborne photogrammetry and lidar for dsm extraction and $3 \mathrm{~d}$ change detection over an urban area-a comparative study," International Journal of Remote Sensing, vol. 34, no. 4, pp. 1087-1110, 2013.

[6] D. Girardeau-Montaut, M. Roux, R. Marc, and G. Thibault, "Change detection on points cloud data acquired with a ground laser scanner," ISPRS Archives, vol. 36-3/W19, pp. 30-35, 2005.

[7] D. Lague, N. Brodu, and J. Leroux, "Accurate 3D comparison of complex topography with terrestrial laser scanner: Application to the rangitikei canyon (NZ), $P \& R S$, vol. 82, pp. 10-26, 2013.

[8] T.H.G. Tran, C. Ressl, and N. Pfeifer, "Integrated change detection and classification in urban areas based on airborne laser scanning point clouds," Sensors, vol. 18, no. 2, pp. 448, 2018.

[9] Z. Zhang, G. Vosselman, M. Gerke, C. Persello, D. Tuia, and M.Y. Yang, "Detecting building changes between airborne laser scanning and photogrammetric data," Remote sensing, vol. 11, no. 20, pp. 2417, 2019. 